\title{
VIETNAM ACADEMY OF SCIENCE AND TECHNOLOGY
}

\section{Vietnam Journal}

\section{ofMECHANICS \\ Volume 36 Number 3}

ISSN 0866-7136

VN INDEX 12.666

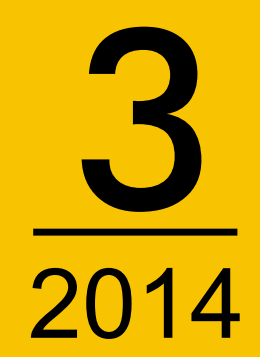


Vietnam Journal of Mechanics, VAST, Vol.36, No. 3 (2014), pp. $201-214$

\title{
AN ANALYTICAL APPROACH TO ANALYZE NONLINEAR DYNAMIC RESPONSE OF ECCENTRICALLY STIFFENED FUNCTIONALLY GRADED CIRCULAR CYLINDRICAL SHELLS SUBJECTED TO TIME DEPENDENT AXIAL COMPRESSION AND EXTERNAL PRESSURE. PART 1: GOVERNING EQUATIONS ESTABLISHMENT
}

\author{
Dao Van Dung ${ }^{1}$, Vu Hoai Nam ${ }^{2, *}$ \\ ${ }^{1}$ Hanoi University of Science, VNU, Vietnam \\ ${ }^{2}$ University of Transport Technology, Hanoi, Vietnam \\ *E-mail: hoainam.vu@utt.edu.vn \\ Received December 25, 2013
}

\begin{abstract}
Based on the classical thin shell theory with the geometrical nonlinearity in von Karman-Donnell sense, the smeared stiffener technique and Galerkin method, this paper deals with the nonlinear dynamic problem of eccentrically stiffened functionally graded circular cylindrical shells subjected to time dependent axial compression and external pressure by analytical approach. The present novelty is that an approximate three-term solution of deflection taking into account the nonlinear buckling shape is chosen, the nonlinear dynamic second-order differential three equations system is established and the frequency-amplitude relation of nonlinear vibration is obtained in explicit form.

Keywords: Functionally graded material, discontinuous reinforcement, buckling, elasticity, analytical modelling.
\end{abstract}

\section{INTRODUCTION}

Many authors studied the static buckling and postbuckling of FGM cylindrical shells subjected to the mechanic and thermal loading. Shen $[1,2]$ investigated the nonlinear postbuckling of thin FGM cylindrical shells and FGM hybrid cylindrical shells in thermal environments under lateral pressure and axial loading, respectively. Bahtui and Eslami [3] investigated the coupled thermo-elasticity of FGM cylindrical shells. Batra and Iaccarino [4] presented the exact solutions for radial deformations of a functionally graded isotropic and incompressible second-order elastic cylinder. Huang and Han [5-7] studied the buckling and postbuckling of un-stiffened FGM cylindrical shells under axial compression, radial pressure and combined axial compression and radial pressure based on the Donnell shell theory and the nonlinear strain-displacement relations and the nonlinear 
three term solution form is used. Sun et al. [8] proposed the accurate symplectic space solutions for thermal buckling of functionally graded cylindrical shells. The postbuckling of shear deformable FGM cylindrical shells surrounded by an elastic medium was studied by Shen [9]. Dung and Hoa [10] investigated the nonlinear torsional buckling and postbuckling of eccentrically stiffened functionally graded thin circular cylindrical shells. Liew et al. [11] studied postbuckling responses of functionally graded cylindrical shells under axial compression and thermal loads. Sofiyev [12] analyzed the buckling of FGM circular shells under combined loads and resting on the Pasternak type elastic foundation. The non-linear static buckling of FGM conical shells which is more general than cylindrical shells, were studied by Sofiyev [13,14]. Torabi et al. [15] studied the linear thermal buckling analysis of truncated hybrid FGM conical shells.

For dynamic analysis of FGM cylindrical shells, Singh et al. [16] investigated torsional vibrations of functionally graded finite cylinders. Darabi et al. [17] presented respectively linear and nonlinear parametric resonance analyses for un-stiffened FGM cylindrical shells. Three-dimensional vibration analysis of fluid-filled orthotropic FGM cylindrical shells was investigated by Chen et al. [18]. Sofiyev and Schnack [19] and Sofiyev [20] obtained critical parameters for un-stiffened cylindrical thin shells under linearly increasing dynamic torsional loading and under a periodic axial impulsive loading by using the Galerkin technique together with Ritz type variation method. Sofiyev [21-24] and Deniz and Sofiyev [25] investigated the vibration and dynamic instability of FGM conical shells. Torsional vibration and stability of functionally graded orthotropic cylindrical shells on elastic foundations is presented by Najafov et al. [26]. Sofiyev and Kuruoglu [27] investigated the torsional vibration and buckling of the cylindrical shell with functionally graded coatings surrounded by an elastic medium. Tornabene and Viola [28] studied free vibration analysis of functionally graded panels and shells of revolution. Huang and Han [29] presented the nonlinear dynamic buckling problems of un-stiffened functionally graded cylindrical shells subjected to time-dependent axial load by using the Budiansky-Roth dynamic buckling criterion [30]. Various effects of the inhomogeneous parameter, loading speed, dimension parameters; environmental temperature rise and initial geometrical imperfection on nonlinear dynamic buckling were discussed. Dynamic analysis of thick short length FGM cylinders was investigated by Asemi et al. [31].

In engineering design, plates and shells are usually reinforced by stiffeners for the benefit of added load carrying capability with a relatively small additional weight. However, the investigation on this field has received comparatively little attention. Najafizadeh et al. [32] have studied linear static buckling of FGM cylindrical shell under axial compression reinforced by FGM stiffeners. Bich et al. [33-36] investigated the nonlinear static and dynamic analysis of FGM plates, cylindrical panels, shallow shells and cylindrical shells with eccentrically homogeneous stiffener system. Dung and Hoa [37] presented an analytical study of nonlinear static buckling and post-buckling analysis of eccentrically stiffened functionally graded circular cylindrical shells under external pressure with FGM stiffeners and approximate three-term solution of deflection taking into account the nonlinear buckling shape.

To best of authors' knowledge, there is no analytical approach on the nonlinear dynamic analysis of stiffened FGM shells subjected to time dependent external pressure and 
axial compression by analytical approach. In addition, the nonlinear three term solution of deflection is popular used to investigate the nonlinear static analysis of shell [5-8 and 37], but there are a mathematical difficulty on the nonlinear dynamic analysis. This paper studies the dynamic behavior of stiffened FGM cylindrical circular shells under mechanic loads. The nonlinear dynamic equations are derived by using the classical shell theory with the nonlinear strain-displacement relation of large deflection, the smeared stiffeners technique and Galerkin method. The three-term solution of deflection is used and the frequency-amplitude relation of nonlinear vibration is obtained in explicit form.

\section{ECCENTRICALLY STIFFENED FGM CYLINDRICAL SHELLS (ES-FGM CYLINDRICAL SHELLS)}

An ES-FGM cylindrical shell as shown in Fig. 1 is assumed to be thin with length $L$, mean radius $R$, reinforced by homogeneous ring and stringer stiffener systems. Stiffener material is similarly designed with Refs. [33-36] is full ceramic if it is located at ceramic-rich surface and is pure-metal if is located at metal-rich surface. The origin of the coordinate 0 locates on the middle plane of the shell, $x, y=R \theta, z$ axes are in the axial, circumferential, and inward radial directions, respectively.
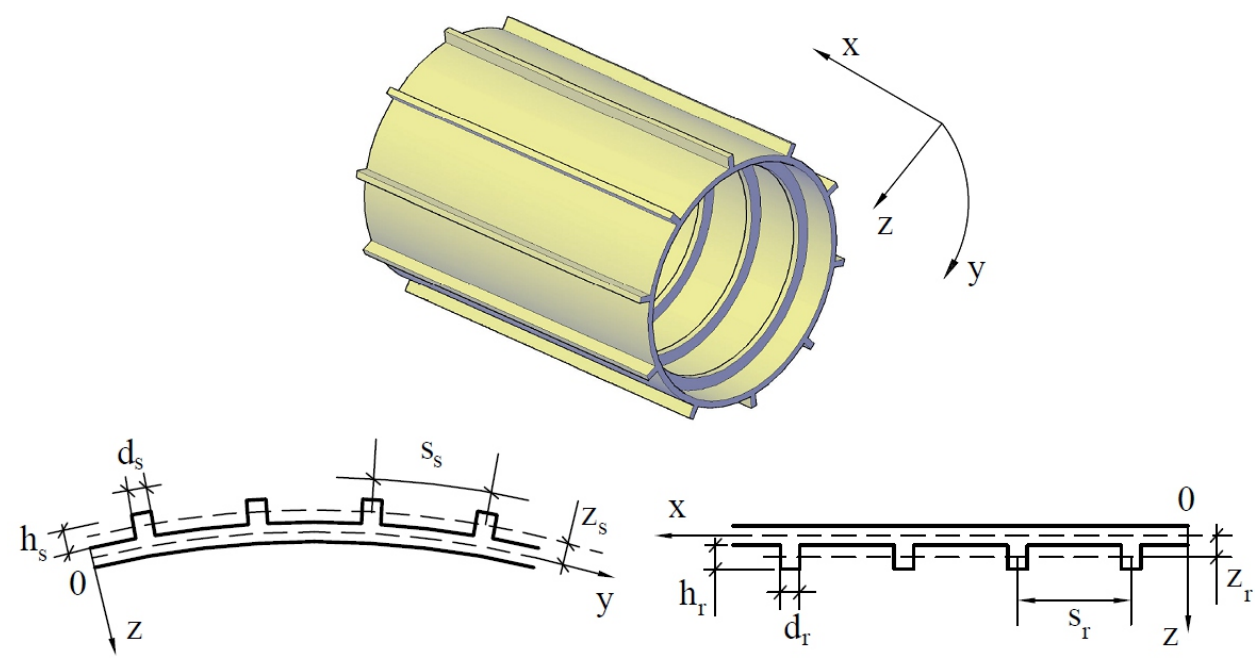

Fig. 1. Geometric and the coordinate system of an eccentrically stiffened FGM cylindrical shell

Functionally graded material is assumed to be made from a mixture of ceramic and metal with the simple power law exponent of volume fraction distribution

$$
V_{c}=V_{c}(z)=\left(\frac{2 z+h}{2 h}\right)^{k}, \quad V_{m}=V_{m}(z)=1-V_{c}(z)
$$

where $h$ is the thickness of shell; $k \geq 0$ is the volume fraction index; $z$ is the thickness coordinate and varies from $-h / 2$ to $h / 2$; the subscripts $m$ and $c$ refer to the metal and ceramic constituents, respectively. 
Effective properties $P r_{e f f}$ of functionally graded material are determined by linear rule of mixture

$$
P r_{e f f}=\operatorname{Pr}_{m}(z) V_{m}(z)+\operatorname{Pr}_{c}(z) V_{c}(z)
$$

The Young's modulus and mass density can be written by according to the mentioned law

$$
\begin{aligned}
& E(z)=E_{m} V_{m}+E_{c} V_{c}=E_{m}+\left(E_{c}-E_{m}\right)\left(\frac{2 z+h}{2 h}\right)^{k}, \\
& \rho(z)=\rho_{m} V_{m}+\rho_{c} V_{c}=\rho_{m}+\left(\rho_{c}-\rho_{m}\right)\left(\frac{2 z+h}{2 h}\right)^{k},
\end{aligned}
$$

and the Poisson's ratio $\nu$ is assumed to be constant for simplicity. According to the von Karman nonlinear strain-displacement relations of cylindrical shell [38], the mid-surface strain components are

$$
\begin{aligned}
& \varepsilon_{x}^{0}=\frac{\partial u}{\partial x}+\frac{1}{2}\left(\frac{\partial w}{\partial x}\right)^{2}, \quad \varepsilon_{y}^{0}=\frac{\partial v}{\partial y}-\frac{w}{R}+\frac{1}{2}\left(\frac{\partial w}{\partial y}\right)^{2}, \\
& \gamma_{x y}^{0}=\frac{\partial u}{\partial y}+\frac{\partial v}{\partial x}+\frac{\partial w}{\partial x} \frac{\partial w}{\partial y}, \quad \chi_{x}=\frac{\partial^{2} w}{\partial x^{2}}, \quad \chi_{y}=\frac{\partial^{2} w}{\partial y^{2}}, \quad \chi_{x y}=\frac{\partial^{2} w}{\partial x \partial y},
\end{aligned}
$$

where $\varepsilon_{x}^{0}$ and $\varepsilon_{y}^{0}$ are normal strains, $\gamma_{x y}^{0}$ is the shear strain at the middle surface of shell, $\chi_{x}, \chi_{y}, \chi_{x y}$ are the change of curvatures and twist of shell, and $u=u(x, y), v=v(x, y)$, $w=w(x, y)$ are displacements along $x, y$ and $z$ axes, respectively. The strains components can be written as the form

$$
\varepsilon_{x}=\varepsilon_{x}^{0}-z \chi_{x}, \quad \varepsilon_{y}=\varepsilon_{y}^{0}-z \chi_{y}, \quad \gamma_{x y}=\gamma_{x y}^{0}-2 z \chi_{x y} .
$$

The deformation compatibility equation is deduced from Eq. (2)

$$
\frac{\partial^{2} \varepsilon_{x}^{0}}{\partial y^{2}}+\frac{\partial^{2} \varepsilon_{y}^{0}}{\partial x^{2}}-\frac{\partial^{2} \gamma_{x y}^{0}}{\partial x \partial y}=-\frac{1}{R} \frac{\partial^{2} w}{\partial x^{2}}+\left(\frac{\partial^{2} w}{\partial x \partial y}\right)^{2}-\frac{\partial^{2} w}{\partial x^{2}} \frac{\partial^{2} w}{\partial y^{2}} .
$$

Hook's stress-strain relation for the shell is presented

$$
\sigma_{x}^{s h}=\frac{E(z)}{1-\nu^{2}}\left(\varepsilon_{x}+\nu \varepsilon_{y}\right), \quad \sigma_{y}^{s h}=\frac{E(z)}{1-\nu^{2}}\left(\varepsilon_{y}+\nu \varepsilon_{x}\right), \quad \tau_{x y}^{s h}=\frac{E(z)}{2(1+\nu)} \gamma_{x y},
$$

and for stiffeners

$$
\sigma_{s}^{s t}=E_{s} \varepsilon_{x}, \quad \sigma_{r}^{s t}=E_{r} \varepsilon_{y},
$$

where $E_{s}, E_{r}$ are Young's modulus of stringer and ring stiffeners, respectively.

Taking into account the contribution of stiffeners by the smeared stiffeners technique and omitting the twist of stiffeners [38] and integrating the stress-strain relations and their moments through the thickness of shell, the force and moment of an ES-FGM cylindrical shell are

$$
\begin{aligned}
& N_{x}=\left(A_{11}+\frac{E_{s} A_{s}}{s_{s}}\right) \varepsilon_{x}^{0}+A_{12} \varepsilon_{y}^{0}-\left(B_{11}+C_{s}\right) \chi_{x}-B_{12} \chi_{y}, \\
& N_{y}=A_{12} \varepsilon_{x}^{0}+\left(A_{22}+\frac{E_{r} A_{r}}{s_{r}}\right) \varepsilon_{y}^{0}-B_{12} \chi_{x}-\left(B_{22}+C_{r}\right) \chi_{y}, \\
& N_{x y}=A_{66} \gamma_{x y}^{0}-2 B_{66} \chi_{x y}
\end{aligned}
$$




$$
\begin{aligned}
& M_{x}=\left(B_{11}+C_{s}\right) \varepsilon_{x}^{0}+B_{12} \varepsilon_{y}^{0}-\left(D_{11}+\frac{E_{s} I_{s}}{s_{s}}\right) \chi_{x}-D_{12} \chi_{y}, \\
& M_{y}=B_{12} \varepsilon_{x}^{0}+\left(B_{22}+C_{r}\right) \varepsilon_{y}^{0}-D_{12} \chi_{x}-\left(D_{22}+\frac{E_{r} I_{r}}{s_{r}}\right) \chi_{y}, \\
& M_{x y}=B_{66} \gamma_{x y}^{0}-2 D_{66} \chi_{x y},
\end{aligned}
$$

where $A_{i j}, B_{i j}, D_{i j}(i, j=1,2,6)$ are extensional, coupling and bending stiffness of the unstiffened FGM cylindrical shell. They are defined as

$$
\begin{array}{llll}
A_{11} & =A_{22}=\frac{E_{1}}{1-\nu^{2}}, & A_{12}=\frac{E_{1} \nu}{1-\nu^{2}}, & A_{66}=\frac{E_{1}}{2(1+\nu)}, \\
B_{11}=B_{22}=\frac{E_{2}}{1-\nu^{2}}, & B_{12}=\frac{E_{2} \nu}{1-\nu^{2}}, & B_{66}=\frac{E_{2}}{2(1+\nu)}, \\
D_{11}=D_{22}=\frac{E_{3}}{1-\nu^{2}}, & D_{12}=\frac{E_{3} \nu}{1-\nu^{2}}, & D_{66}=\frac{E_{3}}{2(1+\nu)},
\end{array}
$$

in which

$$
\begin{aligned}
& E_{1}=\left(E_{m}+\frac{E_{c}-E_{m}}{k+1}\right) h, \quad E_{2}=\frac{\left(E_{c}-E_{m}\right) k h^{2}}{2(k+1)(k+2)} \\
& E_{3}=\left[\frac{E_{m}}{12}+\left(E_{c}-E_{m}\right)\left(\frac{1}{k+3}-\frac{1}{k+2}+\frac{1}{4 k+4}\right)\right] h^{3} \\
& I_{s}=\frac{d_{s} h_{s}^{3}}{12}+A_{s} z_{s}^{2}, \quad I_{r}=\frac{d_{r} h_{r}^{3}}{12}+A_{r} z_{r}^{2} \\
& C_{s}= \pm \frac{E_{s} A_{s} z_{s}}{s_{s}}, \quad C_{r}= \pm \frac{E_{r} A_{r} z_{r}}{s_{r}} \\
& z_{s}=\frac{h_{s}+h}{2}, \quad z_{r}=\frac{h_{r}+h}{2} \\
& s_{s}=\frac{2 \pi R}{n_{s}}, \quad s_{r}=\frac{L}{n_{r}}
\end{aligned}
$$

where

$C_{s}$ and $C_{r}$ are negative for outside stiffeners and positive for inside ones.

$s_{s}$ and $s_{r}$ are the spacing of the stringer and ring stiffeners, respectively.

$d_{s}, h_{s}$ and $d_{r}, h_{r}$ are the width and thickness of the stringer and ring stiffeners, respectively. $A_{s}, A_{r}$ are the cross-section areas of stiffeners.

$I_{s}, I_{r}, z_{s}, z_{r}$ are the second moments of cross section areas and the eccentricities of stiffeners with respect to the middle surface of shell, respectively.

$E_{s}, E_{r}$ are Young's modulus of stringer and ring stiffeners, respectively.

From the Eq. (7), one can obtain inversely

$$
\begin{aligned}
& \varepsilon_{x}^{0}=A_{22}^{*} N_{x}-A_{12}^{*} N_{y}+B_{11}^{*} \chi_{x}+B_{12}^{*} \chi_{y}, \\
& \varepsilon_{y}^{0}=A_{11}^{*} N_{y}-A_{12}^{*} N_{x}+B_{21}^{*} \chi_{x}+B_{22}^{*} \chi_{y}, \\
& \gamma_{x y}^{0}=A_{66}^{*} N_{x y}+2 B_{66}^{*} \chi_{x y},
\end{aligned}
$$


where

$$
\begin{aligned}
& A_{11}^{*}=\frac{1}{\Delta}\left(A_{11}+\frac{E_{s} A_{s}}{s_{s}}\right), A_{22}^{*}=\frac{1}{\Delta}\left(A_{22}+\frac{E_{r} A_{r}}{s_{r}}\right), \\
& A_{12}^{*}=\frac{A_{12}}{\Delta}, A_{66}^{*}=\frac{1}{A_{66}}, \\
& \Delta=\left(A_{11}+\frac{E_{s} A_{s}}{s_{s}}\right)\left(A_{22}+\frac{E_{r} A_{r}}{s_{r}}\right)-A_{12}^{2}, \\
& B_{11}^{*}=A_{22}^{*}\left(B_{11}+C_{s}\right)-A_{12}^{*} B_{12}, \\
& B_{22}^{*}=A_{11}^{*}\left(B_{22}+C_{r}\right)-A_{12}^{*} B_{12}, \\
& B_{12}^{*}=A_{22}^{*} B_{12}-A_{12}^{*}\left(B_{22}+C_{r}\right), \\
& B_{21}^{*}=A_{11}^{*} B_{12}-A_{12}^{*}\left(B_{11}+C_{s}\right), \\
& B_{66}^{*}=\frac{B_{66}}{A_{66}} .
\end{aligned}
$$

Substituting Eq. (11) into Eq. (8) leads to

$$
\begin{aligned}
& M_{x}=B_{11}^{*} N_{x}+B_{21}^{*} N_{y}-D_{11}^{*} \chi_{x}-D_{12}^{*} \chi_{y}, \\
& M_{y}=B_{12}^{*} N_{x}+B_{22}^{*} N_{y}-D_{21}^{*} \chi_{x}-D_{22}^{*} \chi_{y}, \\
& M_{x y}=B_{66}^{*} N_{x y}-2 D_{66}^{*} \chi_{x y},
\end{aligned}
$$

where

$$
\begin{aligned}
& D_{11}^{*}=D_{11}+\frac{E_{s} I_{s}}{s_{s}}-\left(B_{11}+C_{s}\right) B_{11}^{*}-B_{12} B_{21}^{*}, \\
& D_{22}^{*}=D_{22}+\frac{E_{r} I_{r}}{s_{r}}-B_{12} B_{12}^{*}-\left(B_{22}+C_{r}\right) B_{22}^{*}, \\
& D_{12}^{*}=D_{12}-\left(B_{11}+C_{s}\right) B_{12}^{*}-B_{12} B_{22}^{*}, \\
& D_{21}^{*}=D_{12}-B_{12} B_{11}^{*}-\left(B_{22}+C_{r}\right) B_{21}^{*}, \\
& D_{66}^{*}=D_{66}-B_{66} B_{66}^{*} .
\end{aligned}
$$

The nonlinear equations of motion of a thin circular cylindrical shell based on the assumption $u \ll w$ and $v \ll w, \rho_{1} \frac{\partial^{2} u}{\partial t^{2}} \rightarrow 0, \rho_{1} \frac{\partial^{2} v}{\partial t^{2}} \rightarrow 0[17,19,39]$ are given by

$$
\begin{aligned}
& \frac{\partial N_{x}}{\partial x}+\frac{\partial N_{x y}}{\partial y}=0, \\
& \frac{\partial N_{x y}}{\partial x}+\frac{\partial N_{y}}{\partial y}=0, \\
& \frac{\partial^{2} M_{x}}{\partial x^{2}}+2 \frac{\partial^{2} M_{x y}}{\partial x \partial y}+\frac{\partial^{2} M_{y}}{\partial y^{2}}+ \\
& \quad N_{x} \frac{\partial^{2} w}{\partial x^{2}}+2 N_{x y} \frac{\partial^{2} w}{\partial x \partial y}+N_{y} \frac{\partial^{2} w}{\partial y^{2}}+ \\
& +\frac{1}{R} N_{y}+q_{0}=\rho_{1} \frac{\partial^{2} w}{\partial t^{2}}+2 \rho_{1} \mu \frac{\partial w}{\partial t},
\end{aligned}
$$


where $\mu$ is the linear damping coefficient, and

$$
\rho_{1}=\int_{-\frac{h}{2}}^{\frac{h}{2}} \rho(z) d z+\rho_{s} \frac{A_{s}}{s_{s}}+\rho_{r} \frac{A_{r}}{s_{r}}=\left(\rho_{m}+\frac{\rho_{c}-\rho_{m}}{k+1}\right) h+\rho_{s} \frac{A_{s}}{s_{s}}+\rho_{r} \frac{A_{r}}{s_{r}},
$$

with $\rho_{r}=\rho_{m}, \rho_{s}=\rho_{m}$ if stiffeners are full metal and $\rho_{r}=\rho_{c}, \rho_{s}=\rho_{c}$ if stiffeners are full ceramic. The first two of Eq. (15) are identically satisfied by introducing a stress function $\varphi$

$$
N_{x}=\frac{\partial^{2} \varphi}{\partial y^{2}}, \quad N_{y}=\frac{\partial^{2} \varphi}{\partial x^{2}}, \quad N_{x y}=-\frac{\partial^{2} \varphi}{\partial x \partial y} .
$$

Substituting Eq. (11) into Eqs. (4) and (13) and the third of Eq. (15), taking into account Eqs. (2) and (17), we have

$$
\begin{gathered}
A_{11}^{*} \frac{\partial^{4} \varphi}{\partial x^{4}}+\left(A_{66}^{*}-2 A_{12}^{*}\right) \frac{\partial^{4} \varphi}{\partial x^{2} \partial y^{2}}+A_{22}^{*} \frac{\partial^{4} \varphi}{\partial y^{4}}+B_{21}^{*} \frac{\partial^{4} w}{\partial x^{4}}+ \\
+\left(B_{11}^{*}+B_{22}^{*}-2 B_{66}^{*}\right) \frac{\partial^{4} w}{\partial x^{2} \partial y^{2}}+B_{12}^{*} \frac{\partial^{4} w}{\partial y^{4}}+\frac{1}{R} \frac{\partial^{2} w}{\partial x^{2}}-\left[\left(\frac{\partial^{2} w}{\partial x \partial y}\right)^{2}-\frac{\partial^{2} w}{\partial x^{2}} \frac{\partial^{2} w}{\partial y^{2}}\right]=0 \\
\rho_{1} \frac{\partial^{2} w}{\partial t^{2}}+2 \rho_{1} \mu \frac{\partial w}{\partial t}+D_{11}^{*} \frac{\partial^{4} w}{\partial x^{4}}+\left(D_{12}^{*}+D_{21}^{*}+4 D_{66}^{*}\right) \frac{\partial^{4} w}{\partial x^{2} \partial y^{2}}+ \\
+D_{22}^{*} \frac{\partial^{4} w}{\partial y^{4}}-B_{21}^{*} \frac{\partial^{4} \varphi}{\partial x^{4}}-\left(B_{11}^{*}+B_{22}^{*}-2 B_{66}^{*}\right) \frac{\partial^{4} \varphi}{\partial x^{2} \partial y^{2}}-B_{12}^{*} \frac{\partial^{4} \varphi}{\partial y^{4}}- \\
-\frac{1}{R} \frac{\partial^{2} \varphi}{\partial x^{2}}-\frac{\partial^{2} \varphi}{\partial y^{2}} \frac{\partial^{2} w}{\partial x^{2}}+2 \frac{\partial^{2} \varphi}{\partial x \partial y} \frac{\partial^{2} w}{\partial x \partial y}-\frac{\partial^{2} \varphi}{\partial x^{2}} \frac{\partial^{2} w}{\partial y^{2}}-q_{0}=0
\end{gathered}
$$

Eqs. (18) and (19) are a nonlinear governing equation system. They are used to investigate the dynamic characteristics of ES-FGM circular cylindrical shells.

\section{NONLINEAR DYNAMIC BUCKLING ANALYSIS}

Suppose that an ES-FGM cylindrical shell is simply supported and subjected to uniformly distributed pressure of intensity $q_{0}$ and axial compression of intensity $r_{0}$ respectively at its cross-section (in $\mathrm{N} / \mathrm{m}^{2}$ ). The boundary conditions of this study are

$$
w=0, \quad M_{x}=0, \quad N_{x}=-r_{0} h, \quad N_{x y}=0, \quad \text { at } \quad x=0, L .
$$

Assume the buckling mode shape is represented by the popular form [5-8,39], the simply supported boundary condition Eq. (20) is fulfilled on the average sense

$$
w=f_{0}+f_{1} \sin \frac{m \pi x}{L} \sin \frac{n y}{R}+f_{2} \sin ^{2} \frac{m \pi x}{L},
$$

where $f_{0}=f_{0}(t)$ is time dependent unknown uniform deflection of pre-buckling state, $f_{1}=f_{1}(t)$ is time dependent unknown linear buckling deflection, $f_{2}=f_{2}(t)$ is time dependent unknown nonlinear deflection, $m$ is numbers of half waves and $n$ is numbers of full wave in axial and circumferential directions, respectively. 
Substituting Eq. (21) into Eq. (18) and solving obtained equation, leads to

$$
\begin{aligned}
\varphi= & \varphi_{1} \cos \frac{2 m \pi x}{L}+\varphi_{2} \cos \frac{2 n y}{R}-\varphi_{3} \sin \frac{m \pi x}{L} \sin \frac{n y}{R}+ \\
& +\varphi_{4} \sin \frac{3 m \pi x}{L} \sin \frac{n y}{R}-\sigma_{0 y} h \frac{x^{2}}{2}-r_{0} h \frac{y^{2}}{2},
\end{aligned}
$$

where denote

$$
\begin{aligned}
& \varphi_{1}=\frac{n^{2} \lambda^{2}}{32 A_{11}^{*} m^{2} \pi^{2}} f_{1}^{2}-\frac{\left(4 \lambda L-16 B_{21}^{*} m^{2} \pi^{2}\right)}{32 A_{11}^{*} m^{2} \pi^{2}} f_{2}, \quad \varphi_{2}=\frac{m^{2} \pi^{2}}{32 A_{22}^{*} n^{2} \lambda^{2}} f_{1}^{2}, \\
& \varphi_{3}=\frac{B}{A} f_{1}+\frac{m^{2} n^{2} \pi^{2} \lambda^{2}}{A} f_{1} f_{2}, \quad \varphi_{4}=\frac{m^{2} n^{2} \pi^{2} \lambda^{2}}{G} f_{1} f_{2}, \\
& A=A_{11}^{*} m^{4} \pi^{4}+\left(A_{66}^{*}-2 A_{12}^{*}\right) m^{2} n^{2} \pi^{2} \lambda^{2}+A_{22}^{*} n^{4} \lambda^{4}, \\
& B=B_{21}^{*} m^{4} \pi^{4}+\left(B_{11}^{*}+B_{22}^{*}-2 B_{66}^{*}\right) m^{2} n^{2} \pi^{2} \lambda^{2}+B_{12}^{*} n^{4} \lambda^{4}-\frac{L^{2}}{R} m^{2} \pi^{2}, \\
& D=D_{11}^{*} m^{4} \pi^{4}+\left(D_{12}^{*}+D_{21}^{*}+4 D_{66}^{*}\right) m^{2} n^{2} \pi^{2} \lambda^{2}+D_{22}^{*} n^{4} \lambda^{4}, \\
& G=81 A_{11}^{*} m^{4} \pi^{4}+9\left(A_{66}^{*}-2 A_{12}^{*}\right) m^{2} n^{2} \pi^{2} \lambda^{2}+A_{22}^{*} n^{4} \lambda^{4}, \\
& \lambda=\frac{L}{R} .
\end{aligned}
$$

Substituting the expressions (21) and (22) into Eq. (19) and then applying Galerkin method lead to

$$
\begin{aligned}
\sigma_{0 y} h=R q_{0}-R \rho_{1} \frac{d^{2} f_{0}}{d t^{2}}-R \frac{\rho_{1}}{2} \frac{d^{2} f_{2}}{d t^{2}}-2 R \rho_{1} \mu \frac{d f_{0}}{d t}-R \rho_{1} \mu \frac{d f_{2}}{d t}, \\
L^{4} \rho_{1} \frac{d^{2} f_{1}}{d t^{2}}+2 L^{4} \rho_{1} \mu \frac{d f_{1}}{d t}+\left(D+\frac{B^{2}}{A}\right) f_{1}+\left(\frac{m^{4} \pi^{4}}{16 A_{22}^{*}}+\frac{n^{4} \lambda^{4}}{16 A_{11}^{*}}\right) f_{1}^{3}+ \\
+\left[\frac{2 B m^{2} n^{2} \pi^{2} \lambda^{2}}{A}-\frac{n^{2} \lambda^{2}\left(\lambda L-4 B_{21}^{*} m^{2} \pi^{2}\right)}{4 A_{11}^{*}}\right] f_{1} f_{2}+ \\
+\left(\frac{m^{4} n^{4} \pi^{4} \lambda^{4}}{A}+\frac{m^{4} n^{4} \pi^{4} \lambda^{4}}{G}\right) f_{1} f_{2}^{2}-m^{2} \pi^{2} L^{2} h r_{0} f_{1}-\sigma_{0 y} h n^{2} L^{2} \lambda^{2} f_{1}=0 \\
\rho_{1} \frac{d^{2} f_{0}}{d t^{2}}+2 \rho_{1} \mu \frac{d f_{0}}{d t}+\rho_{1} \frac{3}{4} \frac{d^{2} f_{2}}{d t^{2}}+\frac{3}{2} \rho_{1} \mu \frac{d f_{2}}{d t}+ \\
+\left\{\left[4 B_{21}^{*}\left(\frac{m \pi}{L}\right)^{4}-\frac{1}{R}\left(\frac{m \pi}{L}\right)^{2}\right] \frac{n^{2} \lambda^{2}}{16 A_{11}^{*} m^{2} \pi^{2}}+\frac{1}{2} \frac{B}{A}\left(\frac{m \pi}{L}\right)^{2}\left(\frac{n}{R}\right)^{2}\right\} f_{1}^{2}+ \\
+\frac{1}{2} m^{2} n^{2} \pi^{2} \lambda^{2}\left(\frac{m \pi}{L}\right)^{2}\left(\frac{n}{R}\right)^{2}\left(\frac{1}{A}-\frac{1}{G}\right) f_{1}^{2} f_{2}+ \\
+\left\{4 D_{11}^{*}\left(\frac{m \pi}{L}\right)^{4}-\left[4 B_{21}^{*}\left(\frac{m \pi}{L}\right)^{4}-\frac{1}{R}\left(\frac{m \pi}{L}\right)^{2}\right] \frac{\lambda L-4 B_{21}^{*} m^{2} \pi^{2}}{4 A_{11}^{*} m^{2} \pi^{2}}\right\} f_{2}- \\
-r_{0} h\left(\frac{m \pi}{L}\right)^{2} f_{2}+\frac{\sigma_{0 y} h}{R}=q_{0} .
\end{aligned}
$$


For circular cylindrical shell, the circumferential closed condition must be considered as $[29,39]$

$$
\int_{0}^{2 \pi R} \int_{0}^{L} \frac{\partial v}{\partial y} d x d y=\int_{0}^{2 \pi R} \int_{0}^{L}\left[\varepsilon_{y}^{0}+\frac{w}{R}-\frac{1}{2}\left(\frac{\partial w}{\partial y}\right)^{2}\right] d x d y=0 .
$$

From Eqs. (11), (17), (21) and (22), this integral becomes

$$
-2 A_{11}^{*} \sigma_{0 y} h+2 A_{12}^{*} r_{0} h+\frac{1}{R}\left(f_{2}+2 f_{0}\right)-\frac{1}{4}\left(\frac{n}{R}\right)^{2} f_{1}^{2}=0
$$

Eliminating $\sigma_{0 y}$ from Eqs. (25)-(27) and the condition of closed form (29), lead to

$$
\begin{gathered}
\left(\frac{d^{2} f_{0}}{d t^{2}}+2 \mu \frac{d f_{0}}{d t}\right)+\frac{1}{2}\left(\frac{d^{2} f_{2}}{d t^{2}}+2 \mu \frac{d f_{2}}{d t}\right)+\alpha_{11}\left(f_{2}+2 f_{0}\right)-\alpha_{12} f_{1}^{2}-\alpha_{13} q_{0}+\alpha_{14} r_{0}=0 \\
\alpha_{21} f_{1} \\
\left(\frac{d^{2} f_{0}}{d t^{2}}+2 \mu \frac{d f_{0}}{d t}\right)+\left(\frac{d^{2} f_{1}}{d t^{2}}+2 \mu \frac{d f_{1}}{d t}\right)+\frac{\alpha_{21}}{2} f_{1}\left(\frac{d^{2} f_{2}}{d t^{2}}+2 \mu \frac{d f_{2}}{d t}\right)+ \\
+\alpha_{22} f_{1}+\alpha_{23} f_{1} f_{2}+\alpha_{24} f_{1}^{3}+\alpha_{25} f_{1} f_{2}^{2}-\alpha_{26} q_{0} f_{1}-\alpha_{27} r_{0} f_{1}=0 \\
\left(\frac{d^{2} f_{2}}{d t^{2}}+2 \mu \frac{d f_{2}}{d t}\right)+\alpha_{31} f_{1}^{2}+\alpha_{32} f_{1}^{2} f_{2}+\alpha_{33} f_{2}-\alpha_{34} r_{0} f_{2}=0
\end{gathered}
$$

in which

$$
\begin{aligned}
\alpha_{11}=\frac{1}{2 A_{11}^{*} R^{2} \rho_{1}}, \quad \alpha_{12}=\frac{n^{2}}{8 A_{11}^{*} R^{3} \rho_{1}}, \quad \alpha_{13}=\frac{1}{\rho_{1}}, \quad \alpha_{14}=\frac{A_{12}^{*} h}{A_{11}^{*} R \rho_{1}} \\
\alpha_{21}=\frac{R n^{2} \lambda^{2}}{L^{2}}, \quad \alpha_{22}=\frac{1}{L^{4} \rho_{1}}\left(D+\frac{B^{2}}{A}\right), \\
\alpha_{23}=\frac{1}{L^{4} \rho_{1}}\left[\frac{2 B m^{2} n^{2} \pi^{2} \lambda^{2}}{A}-\frac{n^{2} \lambda^{2}\left(\lambda L-4 B_{21}^{*} m^{2} \pi^{2}\right)}{4 A_{11}^{*}}\right], \\
\alpha_{24}=\frac{1}{L^{4} \rho_{1}}\left(\frac{m^{4} \pi^{4}}{16 A_{22}^{*}}+\frac{n^{4} \lambda^{4}}{16 A_{11}^{*}}\right), \quad \alpha_{25}=\frac{1}{L^{4} \rho_{1}}\left(\frac{m^{4} n^{4} \pi^{4} \lambda^{4}}{A}+\frac{m^{4} n^{4} \pi^{4} \lambda^{4}}{G}\right), \\
\alpha_{26}=\frac{R n^{2} \lambda^{2}}{L^{2} \rho_{1}}, \quad \alpha_{27}=\frac{m^{2} \pi^{2} h}{L^{2} \rho_{1}}, \\
\alpha_{31}=\frac{1}{\rho_{1}}\left\{\left[4 B_{21}^{*}\left(\frac{m \pi}{L}\right)^{4}-\frac{1}{R}\left(\frac{m \pi}{L}\right)^{2}\right] \frac{n^{2} \lambda^{2}}{4 A_{11}^{*} m^{2} \pi^{2}}+2 \frac{B}{A}\left(\frac{m \pi}{L}\right)^{2}\left(\frac{n}{R}\right)^{2}\right\} \\
\alpha_{32}=\frac{2}{\rho_{1}} m^{2} n^{2} \pi^{2} \lambda^{2}\left(\frac{m \pi}{L}\right)^{2}\left(\frac{n}{R}\right)^{2}\left(\frac{1}{A}-\frac{1}{G}\right), \\
\alpha_{33}=\frac{1}{\rho_{1}}\left\{16 D_{11}^{*}\left(\frac{m \pi}{L}\right)^{4}-\left[4 B_{21}^{*}\left(\frac{m \pi}{L}\right)^{4}-\frac{1}{R}\left(\frac{m \pi}{L}\right)^{2}\right] \frac{\lambda L-4 B_{21}^{*} m^{2} \pi^{2}}{A_{11}^{*} m^{2} \pi^{2}}\right\} \\
\alpha_{34}=\frac{4}{\rho_{1}} h\left(\frac{m \pi}{L}\right)^{2} \cdot
\end{aligned}
$$


Simplifying Eqs. (30)-(32), we have

$$
\begin{aligned}
& \left(\frac{d^{2} f_{0}}{d t^{2}}+2 \mu \frac{d f_{0}}{d t}\right)+\beta_{11} f_{0}-\beta_{12} f_{1}^{2}-\beta_{13} f_{1}^{2} f_{2}+\beta_{14} f_{2}+\beta_{15} r_{0} f_{2}-\alpha_{13} q_{0}+\alpha_{14} r_{0}=0 \\
& \left(\frac{d^{2} f_{1}}{d t^{2}}+2 \mu \frac{d f_{1}}{d t}\right)+\alpha_{22} f_{1}+\beta_{21} f_{1} f_{0}+\beta_{22} f_{1} f_{2}+\alpha_{25} f_{1} f_{2}^{2}+\beta_{23} f_{1}^{3}-\beta_{24} f_{1} r_{0}=0 \\
& \left(\frac{d^{2} f_{2}}{d t^{2}}+2 \mu \frac{d f_{2}}{d t}\right)+\alpha_{31} f_{1}^{2}+\alpha_{32} f_{1}^{2} f_{2}+\alpha_{33} f_{2}-\alpha_{34} r_{0} f_{2}=0
\end{aligned}
$$

where

$$
\begin{gathered}
\beta_{11}=2 \alpha_{11}, \beta_{12}=\frac{1}{2} \alpha_{31}+\alpha_{12}, \beta_{13}=\frac{1}{2} \alpha_{32}, \beta_{14}=\alpha_{11}-\frac{1}{2} \alpha_{33}, \beta_{15}=\frac{1}{2} \alpha_{34}, \\
\beta_{21}=-\beta_{11} \alpha_{21}, \quad \beta_{22}=-\beta_{14} \alpha_{21}-\alpha_{33} \frac{\alpha_{21}}{2}+\alpha_{23}, \\
\beta_{23}=\beta_{12} \alpha_{21}-\alpha_{31} \frac{\alpha_{21}}{2}+\alpha_{24}, \quad \beta_{24}=\alpha_{27}+\alpha_{14} \alpha_{21} .
\end{gathered}
$$

Denoting $f=w_{\max }$, from Eq. (21), the maximal deflection of the shells

$$
f=f_{0}+f_{1}+f_{2}
$$

locates at $x=\frac{i L}{2 m}, y=\frac{j \pi R}{2 n}$ where $i, j$ are odd integer numbers. Note that $f_{0}=f_{0}(t)$, $f_{1}=f_{1}(t), f_{2}=f_{2}(t)$ and $f=f(t)$ in Eq. (41).

From Eqs. (36)-(38) and (41), the effects of input parameters on the dynamic response of shells are investigated.

\subsection{Nonlinear vibration analysis}

This section considers an ES-FGM cylindrical thin shell under uniformly lateral pressure $q_{0}=Q \sin \Omega t$ and $r_{0}=0$, Eqs. (36)-(38) become

$$
\begin{aligned}
& \left(\frac{d^{2} f_{0}}{d t^{2}}+2 \mu \frac{d f_{0}}{d t}\right)+\beta_{11} f_{0}-\beta_{12} f_{1}^{2}-\beta_{13} f_{1}^{2} f_{2}+\beta_{14} f_{2}-\alpha_{13} Q \sin \Omega t=0 \\
& \left(\frac{d^{2} f_{1}}{d t^{2}}+2 \mu \frac{d f_{1}}{d t}\right)+\alpha_{22} f_{1}+\beta_{21} f_{1} f_{0}+\beta_{22} f_{1} f_{2}+\alpha_{25} f_{1} f_{2}^{2}+\beta_{23} f_{1}^{3}=0 \\
& \left(\frac{d^{2} f_{2}}{d t^{2}}+2 \mu \frac{d f_{2}}{d t}\right)+\alpha_{31} f_{1}^{2}+\alpha_{32} f_{1}^{2} f_{2}+\alpha_{33} f_{2}=0
\end{aligned}
$$

where $Q$ is excitation force and $\Omega$ is excitation frequency. From these equations, nonlinear response of ES-FGM shell is investigated by using the fourth order Runge-Kutta iteration method.

It is difficult to determine the fundamental frequencies of natural vibration, frequencyamplitude relation of nonlinear vibration of shell. In this paper, ones can be investigated by ignoring the uniform buckling shape and nonlinear buckling shape, Eq. (31) becomes

$$
\left(\frac{d^{2} f_{1}}{d t^{2}}+2 \mu \frac{d f_{1}}{d t}\right)+\alpha_{22} f_{1}+\alpha_{24} f_{1}^{3}-\alpha_{26} f_{1} Q \sin \Omega t=0
$$


For the free and linear vibration without damping, the Eq. (45) becomes

$$
\frac{d^{2} f_{1}}{d t^{2}}+\alpha_{22} f_{1}=0
$$

The fundamental frequency of natural vibration can be determined by

$$
\omega_{m n}=\sqrt{\alpha_{22}} .
$$

where $\omega_{m n}$ is fundamental frequency of natural vibration of shell.

Using the solution $f_{1}(t)=\eta \sin (\Omega t)$ and applying the procedure like Galerkin method to Eq. (45), the frequency-amplitude relation of nonlinear vibration is obtained

$$
\Omega^{2}-\frac{4}{\pi} \mu \Omega=\alpha_{22}+\frac{3}{4} \alpha_{24} \eta^{2}-\frac{8}{3 \pi} \alpha_{26} Q
$$

By introducing the non-dimension frequency parameter $\xi=\frac{\Omega}{\omega_{m n}}$, Eq. (48) becomes

$$
\xi^{2}-\frac{4 \mu}{\pi \omega_{m n}} \xi=1+\frac{3}{4} \frac{\alpha_{24}}{\omega_{m n}^{2}} \eta^{2}-\frac{8}{3 \pi} \frac{\alpha_{26}}{\omega_{m n}^{2}} Q
$$

The frequency-amplitude relation of nonlinear free vibration $Q=0$ is determined by

$$
\xi^{2}-\frac{4 \mu}{\pi \omega_{m n}} \xi=1+\frac{3}{4} \frac{\alpha_{24}}{\omega_{m n}^{2}} \eta^{2} .
$$

\subsection{Buckling analysis}

\subsubsection{Linear static buckling analysis of ES-FGM cylindrical shells}

Omitting the linear damping, uniform buckling shape, nonlinear buckling shape and putting $\dot{f}_{1}=0, \ddot{f}_{1}=0$, and taking $f_{1} \neq 0$ Eq. (31) becomes

$$
\alpha_{22}+\alpha_{24} f_{1}^{2}-\alpha_{26} q_{0}-\alpha_{27} r_{0}=0 .
$$

By ignoring the nonlinear term of $f_{1}$ and $r_{0}=0$ in Eq. (51), the linear upper static buckling load of ES-FGM cylindrical shells under only external pressure can be determined by

$$
q_{s b u}=\frac{\alpha_{22}}{\alpha_{26}} .
$$

Similarly, the linear upper static buckling load of ES-FGM cylindrical shells under only axial compression $\left(q_{0}=0\right)$ leads to

$$
r_{s b u}=\frac{\alpha_{22}}{\alpha_{27}}
$$

From Eqs. (52)-(53), the linear static critical buckling loads of shells are determined by $r_{s c r}=\min r_{s b u} \forall(m, n)$ and $q_{s c r}=\min q_{s b u} \forall(m, n)$. 


\subsubsection{Dynamic buckling analysis of ES-FGM cylindrical shells}

The nonlinear dynamic critical buckling analysis of ES-FGM circular cylindrical shells based on Eqs. (36)-(38), is investigated for two load types as follows.

Firstly, ES-FGM cylindrical shell is subjected to only lateral pressure varying as linear function of time $q_{0}=c_{q} t$ in which $c_{q}\left(\mathrm{~N} / \mathrm{m}^{2} \mathrm{~s}\right)$ is the loading speed of external pressure.

Secondly, ES-FGM cylindrical shell is subjected to only axial compression varying as linear function of time $r_{0}=c_{r} t$ where $c_{r}\left(\mathrm{~N} / \mathrm{m}^{2} \mathrm{~s}\right)$ is the loading speed of axial compression.

Eqs. (36)-(38) are the nonlinear second-order differential three equations system. This equation system may be numerically solved.

\section{CONCLUSIONS}

A formulation of governing equations of eccentrically stiffened functionally graded circular cylindrical thin shells subjected to time dependent axial compression and external pressure based upon the classical shell theory and the smeared stiffeners technique with von Karman-Donnell nonlinear terms is proposed in this paper. An approximate threeterm solution of deflection taking into account the nonlinear buckling shape is used. The nonlinear dynamic equations of ES-FGM circular cylindrical shells are obtained by using the Galerkin method. Fundamental frequency of natural vibration, frequency-amplitude relation of nonlinear vibration and upper static buckling loads are obtained in explicit forms. Dynamic responses will be numerically investigated and nonlinear dynamic buckling loads will be determined by applying Budiansky-Roth criterion in next part.

\section{ACKNOWLEDGEMENTS}

This research is funded by Vietnam National Foundation for Science and Technology Development (NAFOSTED) under grant number 107.02-2013.02.

\section{REFERENCES}

[1] H. S. Shen. Postbuckling analysis of pressure-loaded functionally graded cylindrical shells in thermal environments. Engineering Structures, 25, (4), (2003), pp. 487-497.

[2] H. S. Shen. Postbuckling of axially loaded FGM hybrid cylindrical shells in thermal environments. Composites Science and Technology, 65, (11), (2005), pp. 1675-1690.

[3] A. Bahtui and M. Eslami. Coupled thermoelasticity of functionally graded cylindrical shells. Mechanics Research Communications, 34, (1), (2007), pp. 1-18.

[4] R. Batra and G. Iaccarino. Exact solutions for radial deformations of a functionally graded isotropic and incompressible second-order elastic cylinder. International Journal of NonLinear Mechanics, 43, (5), (2008), pp. 383-398.

[5] H. Huang and Q. Han. Buckling of imperfect functionally graded cylindrical shells under axial compression. European Journal of Mechanics-A/Solids, 27, (6), (2008), pp. 1026-1036.

[6] H. Huang and Q. Han. Nonlinear elastic buckling and postbuckling of axially compressed functionally graded cylindrical shells. International Journal of Mechanical Sciences, 51, (7), (2009), pp. 500-507. 
[7] H. Huang and Q. Han. Nonlinear buckling and postbuckling of heated functionally graded cylindrical shells under combined axial compression and radial pressure. International Journal of Non-Linear Mechanics, 44, (2), (2009), pp. 209-218.

[8] J. Sun, X. Xu, and C. Lim. Accurate symplectic space solutions for thermal buckling of functionally graded cylindrical shells. Composites Part B: Engineering, 55, (2013), pp. 208214.

[9] H. S. Shen. Postbuckling of shear deformable FGM cylindrical shells surrounded by an elastic medium. International Journal of Mechanical Sciences, 51, (5), (2009), pp. 372-383.

[10] D. V. Dung and L. K. Hoa. Research on nonlinear torsional buckling and post-buckling of eccentrically stiffened functionally graded thin circular cylindrical shells. Composites Part B: Engineering, 51, (2013), pp. 300-309.

[11] K. M. Liew, X. Zhao, and Y. Lee. Postbuckling responses of functionally graded cylindrical shells under axial compression and thermal loads. Composites Part B: Engineering, 43, (3), (2012), pp. 1621-1630.

[12] A. H. Sofiyev. Buckling analysis of FGM circular shells under combined loads and resting on the Pasternak type elastic foundation. Mechanics Research Communications, 37, (6), (2010), pp. 539-544.

[13] A. H. Sofiyev. Non-linear buckling behavior of FGM truncated conical shells subjected to axial load. International Journal of Non-Linear Mechanics, 46, (5), (2011), pp. 711-719.

[14] A. H. Sofiyev. Influence of the initial imperfection on the non-linear buckling response of FGM truncated conical shells. International Journal of Mechanical Sciences, 53, (9), (2011), pp. $753-761$.

[15] J. Torabi, Y. Kiani, and M. Eslami. Linear thermal buckling analysis of truncated hybrid FGM conical shells. Composites Part B: Engineering, 50, (2013), pp. 265-272.

[16] B. Singh, J. Rokne, and R. Dhaliwal. Torsional vibrations of functionally graded finite cylinders. Meccanica, 41, (4), (2006), pp. 459-470.

[17] M. Darabi, M. Darvizeh, and A. Darvizeh. Non-linear analysis of dynamic stability for functionally graded cylindrical shells under periodic axial loading. Composite Structures, 83, (2), (2008), pp. 201-211.

[18] W. Chen, Z. Bian, and H. Ding. Three-dimensional vibration analysis of fluid-filled orthotropic FGM cylindrical shells. International Journal of Mechanical Sciences, 46, (1), (2004), pp. 159171.

[19] A. H. Sofiyev and E. Schnack. The stability of functionally graded cylindrical shells under linearly increasing dynamic torsional loading. Engineering Structures, 26, (10), (2004), pp. 13211331.

[20] A. H. Sofiyev. The stability of compositionally graded ceramic-metal cylindrical shells under aperiodic axial impulsive loading. Composite Structures, 69, (2), (2005), pp. 247-257.

[21] A. H. Sofiyev. Dynamic buckling of functionally graded cylindrical thin shells under nonperiodic impulsive loading. Acta Mechanica, 165, (3-4), (2003), pp. 151-163.

[22] A. H. Sofiyev. The stability of functionally graded truncated conical shells subjected to aperiodic impulsive loading. International Journal of Solids and Structures, 41, (13), (2004), pp. 3411-3424.

[23] A. H. Sofiyev. The vibration and stability behavior of freely supported FGM conical shells subjected to external pressure. Composite Structures, 89, (3), (2009), pp. 356-366.

[24] A. H. Sofiyev. The non-linear vibration of FGM truncated conical shells. Composite Structures, 94, (7), (2012), pp. 2237-2245. 
[25] A. Deniz and A. Sofiyev. The nonlinear dynamic buckling response of functionally graded truncated conical shells. Journal of Sound and Vibration, 332, (4), (2013), pp. 978-992.

[26] A. Najafov, A. Sofiyev, and N. Kuruoglu. Torsional vibration and stability of functionally graded orthotropic cylindrical shells on elastic foundations. Meccanica, 48, (4), (2013), pp. 829-840.

[27] A. H. Sofiyev and N. Kuruoglu. Torsional vibration and buckling of the cylindrical shell with functionally graded coatings surrounded by an elastic medium. Composites Part B: Engineering, 45, (1), (2013), pp. 1133-1142.

[28] F. Tornabene and E. Viola. Free vibration analysis of functionally graded panels and shells of revolution. Meccanica, 44, (3), (2009), pp. 255-281.

[29] H. Huang and Q. Han. Nonlinear dynamic buckling of functionally graded cylindrical shells subjected to time-dependent axial load. Composite Structures, 92, (2), (2010), pp. 593-598.

[30] B. Budiansky and R. S. Roth. Axisymmetric dynamic buckling of clamped shallow spherical shells. NASA technical note D_510, (1962), pp. 597-606.

[31] K. Asemi, M. Akhlaghi, and M. Salehi. Dynamic analysis of thick short length FGM cylinders. Meccanica, 47, (6), (2012), pp. 1441-1453.

[32] M. Najafizadeh, A. Hasani, and P. Khazaeinejad. Mechanical stability of functionally graded stiffened cylindrical shells. Applied Mathematical Modelling, 33, (2), (2009), pp. 1151-1157.

[33] D. H. Bich, V. H. Nam, and N. T. Phuong. Nonlinear postbuckling of eccentrically stiffened functionally graded plates and shallow shells. Vietnam Journal of Mechanics, 33, (3), (2011), pp. 131-147.

[34] D. H. Bich, D. V. Dung, and V. H. Nam. Nonlinear dynamical analysis of eccentrically stiffened functionally graded cylindrical panels. Composite Structures, 94, (8), (2012), pp. 2465-2473.

[35] D. H. Bich, D. V. Dung, and V. H. Nam. Nonlinear dynamic analysis of eccentrically stiffened imperfect functionally graded doubly curved thin shallow shells. Composite Structures, 96, (2013), pp. 384-395.

[36] D. H. Bich, D. V. Dung, V. H. Nam, and N. T. Phuong. Nonlinear static and dynamic buckling analysis of imperfect eccentrically stiffened functionally graded circular cylindrical thin shells under axial compression. International Journal of Mechanical Sciences, 74, (2013), pp. 190200.

[37] D. V. Dung and L. K. Hoa. Nonlinear buckling and post-buckling analysis of eccentrically stiffened functionally graded circular cylindrical shells under external pressure. Thin-Walled Structures, 63, (2013), pp. 117-124.

[38] D. O. Brush and B. O. Almroth. Buckling of bars, plates, and shells, Vol. 6. McGraw-Hill New York, (1975).

[39] A. S. Volmir. Non-linear dynamics of plates and shells. Science Edition M, (1972). 
VIETNAM ACADEMY OF SCIENCE AND TECHNOLOGY

VIETNAM JOURNAL OF MECHANICS VOLUME 36, N. 3, 2014

\section{CONTENTS}

Pages

1. N. D. Anh, V. L. Zakovorotny, D. N. Hao, Van der Pol-Duffing oscillator under combined harmonic and random excitations.

2. Pham Hoang Anh, Fuzzy analysis of laterally-loaded pile in layered soil.

3. Dao Huy Bich, Nguyen Dang Bich, On the convergence of a coupling successive approximation method for solving Duffing equation.

4. Dao Van Dung, Vu Hoai Nam, An analytical approach to analyze nonlinear dynamic response of eccentrically stiffened functionally graded circular cylindrical shells subjected to time dependent axial compression and external pressure. Part 1: Governing equations establishment.

5. Manh Duong Phung, Thuan Hoang Tran, Quang Vinh Tran, Stable control of networked robot subject to communication delay, packet loss, and out-oforder delivery.

6. Phan Anh Tuan, Vu Duy Quang, Estimation of car air resistance by CFD method. 